\title{
Glutathione Peroxidase Level in Patients with Vitiligo: A Meta-Analysis
}

\author{
Bi-huan Xiao, ${ }^{1}$ Meihui Shi, ${ }^{1}$ Hongqiang Chen, ${ }^{2}$ Shaoshan Cui, ${ }^{3}$ Yan Wu, \\ Xing-Hua Gao, ${ }^{1}$ and Hong-Duo Chen ${ }^{1}$ \\ ${ }^{1}$ Department of Dermatology, No. 1 Hospital of China Medical University, Shenyang 110001, China \\ ${ }^{2}$ Department of General Surgery, Shandong Qianfoshan Hospital, Jinan 250014, China \\ ${ }^{3}$ Department of Dermatology, The First Affiliated Hospital of Dalian Medical University, Dalian 116011, China \\ Correspondence should be addressed to Yan Wu; jlwuyan@126.com and Xing-Hua Gao; gaobarry@hotmail.com
}

Received 25 December 2015; Revised 18 March 2016; Accepted 5 April 2016

Academic Editor: Davinder Parsad

Copyright (C) 2016 Bi-huan Xiao et al. This is an open access article distributed under the Creative Commons Attribution License, which permits unrestricted use, distribution, and reproduction in any medium, provided the original work is properly cited.

\begin{abstract}
Abnormality of glutathione peroxidase (GPx) is involved in the etiology and pathogenesis of vitiligo. However, the results were controversial. Aim. The purpose of this meta-analysis is to compare the levels of GPx between vitiligo patients and healthy controls. Methods. Relevant published articles were searched according to eligibility criteria. A meta-analysis was conducted to pool estimates of the standardized mean difference (SMD) with 95\% confidence interval (CI). Results. Twenty-three studies with a total of 1076 vitiligo patients and 770 healthy controls were included. The pooled meta-analysis showed that patients with vitiligo had equivalent levels of GPx with the healthy controls ( $\mathrm{SMD}=-0.47,95 \% \mathrm{CI}:-1.03$ to 0.08 , and $p=0.095$ ). Further subgroup analysis showed that the GPx levels of Asian patients or segmental vitiligo patients were, respectively, lower than those of healthy controls (Asian: SMD = $-0.47,95 \%$ CI: -1.08 to 0.14 , and $p=0.001$; segmental: $\mathrm{SMD}=-3.59,95 \% \mathrm{CI}:-6.38$ to -0.80 , and $p=0.012$ ). Furthermore, the GPx levels in serum/plasma were significantly decreased in either stable or active vitiligo patients, comparing to healthy controls (stable: SMD $=-2.01,95 \%$ CI: -3.52 to -0.49 , and $p=0.009$; active: SMD $=-2.34,95 \%$ CI: -4.07 to -0.61 , and $p=0.008)$. Conclusion. This meta-analysis showed a significant association between low GPx level and vitiligo.
\end{abstract}

\section{Introduction}

Vitiligo is an idiopathic, acquired pigmentation disorder of skin and/or mucosa, with clinical manifestations of porcelain white patches. It is considered to be a multifactorial and polygenic disease caused by the destruction of melanocytes [1]. Amongst others, oxidative stress is considered to be one of the causative factors in the pathogenesis of vitiligo [2].

Glutathione peroxidase (GPx) is the general name of an enzyme family with peroxidase activity. It protects cells from oxidative damage through decreasing lipid hydroperoxides to their corresponding alcohols or reducing free hydrogen peroxide to water [3]. In vitiligo, many researches about this antioxidant marker have been sought, but the conclusions were conflicting. Some researchers reported elevated level, whereas others showed no change or reduced level. Due to the inconsistent results, we do the meta-analysis to clarify the GPx level in vitiligo patients.

\section{Materials and Methods}

2.1. Search Strategy. The PubMed, Cochrane Library, Web of Science, Chinese National Knowledge Infrastructure (CNKI), and Wan Fang Med Online were searched by two independent investigators using the search terms ("vitiligo") and ("glutathione peroxidase" or "GPx" or "GSH-Px" or "oxidant" or "antioxidant"). Additional potential relevant articles were further retrieved through a manual search of references from original reports. The research dated from the earliest time to December 2015.

2.2. Eligibility Criteria and Excluded Studies. We sought existing studies published in English or Chinese. Articles 
were included in this meta-analysis if (1) the case group consisted of vitiligo patients and the control group included healthy individuals and (2) the outcome measures reported quantitative GPx level (mean \pm standard deviation). After reading the title and abstract, we excluded a study if it (1) was an animal or in vitro experiment, (2) was a case report or a review, and (3) consisted of duplicate data with other study. All studies were deliberately reviewed by two investigators to decide whether to be included.

2.3. Data Extraction. Two investigators independently screened studies for eligible articles. The following items including the first author, year of publication, nation, sample size, sources, test method of GPx, GPx estimated value, unit, type, and stage of vitiligo were extracted. If there were discrepancies, they would reach a consensus through discussion and reexamination or seeking help to a third investigator.

2.4. Quality Assessment. To estimate the quality of included studies, the Newcastle-Ottawa Scale (NOS) criteria were used by two investigators independently [4]. The NOS criteria were scored based on three aspects: (1) subject selection, $0 \sim 4$, (2) comparability of subject, $0 \sim 2$, and (3) clinical outcome, $0 \sim 3$. Total NOS scores range from 0 (the lowest) to 9 (the highest). Any discrepancy between the two investigators on NOS scores of the enrolled studies was resolved by discussion or consultation with a third investigator.

2.5. Statistical Analysis. The standard mean difference (SMD) for the effect and corresponding 95\% CIs were calculated from the original data of the appropriate studies in fixed effects model (Mantel-Haenszel method) or random-effects model (DerSimonian and Laird method). The random-effects model was applied when heterogeneity existed among studies, while the fixed effects model was applied when there was no statistical heterogeneity. In order to test for comparability, heterogeneity across the included studies was evaluated by Cochran $Q$ test and $I^{2}$ test [5]. Subgroup meta-analyses were conducted according to race (Caucasian versus Asian), stage (active or stable), type (segmental or nonsegmental), or source of sample. The funnel plot was constructed to assess the effect of publication bias on the validity of the estimates. The symmetry of the funnel plot was further evaluated by Egger's linear regression test [6]. All tests were two-sided, and a $p$ value of $<0.05$ was regarded as statistically significant. Stata version 11.0 software (StataCorp., College Station, TX, USA) was performed for statistical analysis.

\section{Results}

3.1. Eligible Studies. We identified 215 studies according to search strategy as shown in Figure 1. After carefully reviewing and screening, 23 articles [3,7-28] were finally included in the meta-analysis. The characteristic and methodological qualities of these studies were shown in Table 1 . The overall study quality ranged from 5 to 7 stars. Of the 23 studies, the sample resource of 20 studies was either serum, plasma, erythrocyte, blood, skin, or blister fluid, and other 3 studies, respectively, tested the GPx level in two sample sources. So the total number of comparisons used in the meta-analysis was 26. The race of all included studies was Caucasian or Asian population. The levels of GPx stratified by sample sources and races were listed in Table 2.

3.2. The Levels of GPX in Vitiligo Patients and Healthy Controls. Random-effects model was applied to the pooled meta-analysis, as statistical heterogeneity existed among studies $\left(\chi^{2}=741.66, p=0.000\right.$, and $\left.I^{2}=96.6 \%\right)$. The results indicated that patients with vitiligo had equivalent levels of GPx with the healthy controls (SMD $=-0.47,95 \% \mathrm{CI}:-1.03$ to 0.08 , and $p=0.095$ ) (Figure 2).

Further subgroup analysis stratified by sample sources indicated that vitiligo patients had higher GPx levels than controls in skin (SMD $=1.49,95 \%$ CI: 0.06 to 2.91 , and $p=$ 0.041 ) and lower GPx levels than controls in blood (SMD = $-1.06,95 \% \mathrm{CI}:-2.06$ to -0.06 , and $p=0.038)$. No difference was seen in the source of serum (SMD $=-1.24,95 \% \mathrm{CI}:-2.79$ to 0.31 , and $p=0.117$ ), plasma (SMD $=-0.05,95 \% \mathrm{CI}:-1.43$ to 1.34 , and $p=0.948$ ), erythrocyte (SMD $=-0.97,95 \%$ CI: -1.94 to 0.00 , and $p=0.050$ ), or blister fluid (SMD = $-0.29,95 \%$ CI: -1.56 to 0.98 , and $p=0.657$ ) (Figure $3(\mathrm{a})$ ). The analysis stratified by race indicated that vitiligo patients in Asian populations had lower GPx levels than controls $(\mathrm{SMD}=-0.47,95 \% \mathrm{CI}:-1.08$ to 0.14 , and $p=0.001)$, but no difference was shown in Caucasian populations (SMD = $0.259,95 \%$ CI: -0.28 to 0.80 , and $p=0.346$ ) (Figure $3(b)$ ). Five articles were included in the subgroup analyses stratified by stage and sample source of serum/plasma (Table 3 ). The results indicated that the vitiligo patients at either stable stage or active stage had lower GPx levels in serum/plasma compared to controls (stable: SMD $=-2.01,95 \% \mathrm{CI}:-3.52$ to -0.49 , and $p=0.009$; active: $\mathrm{SMD}=-2.34,95 \% \mathrm{CI}:-4.07$ to -0.61 , and $p=0.008$ ) (Figures 4(a) and 4(b)). No significant difference was observed between stable stage and active stage $(\mathrm{SMD}=0.50,95 \% \mathrm{CI}:-0.02$ to 1.01 , and $p=0.058)$. Three articles were included in the subgroup analyses stratified by vitiligo type (Table 4). Segmental vitiligo patients had lower GPx levels compared to controls $(\mathrm{SMD}=-3.59,95 \% \mathrm{CI}$ : -6.38 to -0.80 , and $p=0.012$ ). No significant difference was observed between nonsegmental vitiligo patients and controls (SMD $=-2.81,95 \% \mathrm{CI}:-5.71$ to 0.10 , and $p=0.058$ ) or between segmental and nonsegmental vitiligo patients $(\mathrm{SMD}=-0.18,95 \% \mathrm{CI}:-0.47$ to 0.11 , and $p=0.230)$.

3.3. Metaregression and Sensitivity Analyses. Univariate and multivariate metaregression analyses were used to explore possible sources of heterogeneity. The results showed that race could be the major source of heterogeneity (Table 5). The results of sensitivity analysis suggested that no individual studies significantly affected the pooled results, indicating a statistically robust result (Figure 5).

3.4. Publication Bias. We used Egger's test to estimate the possibility of publication bias. The results showed that there 
TABLE 1: Characteristic and methodological qualities of included studies.

\begin{tabular}{|c|c|c|c|c|c|c|c|c|}
\hline Study & Nation & $\begin{array}{c}\text { Number of } \\
\text { participants } \\
\text { (patients/controls) }\end{array}$ & Source & Test method & Unit & Type & Stage & $\begin{array}{l}\text { NOS } \\
\text { score }\end{array}$ \\
\hline Zedan et al. [3] & Egypt & $60 / 30$ & Serum & 1 & $\mathrm{U} / \mathrm{L}$ & $\begin{array}{c}\text { Generalized, } \\
\text { localized }\end{array}$ & $\begin{array}{l}\text { Stable, } \\
\text { active }\end{array}$ & 7 \\
\hline Barikbin et al. [7] & Iran & $60 / 45$ & Serum & 2 & $\mathrm{U} / \mathrm{L}$ & Vulgaris & Active & 5 \\
\hline Ozturk et al. [8] & Turkey & $30 / 30$ & Serum & 4 & $\begin{array}{c}\mathrm{U} / \mathrm{mg} \\
\text { protein }\end{array}$ & Generalized & Stable & 5 \\
\hline Zhao et al. [9] & China & $40 / 10$ & Serum & - & - & - & $\begin{array}{l}\text { Stable, } \\
\text { active }\end{array}$ & 5 \\
\hline Chen et al. [10] & China & $132 / 50$ & Serum & 3 & $\mathrm{U} / \mathrm{L}$ & $\begin{array}{l}\text { Vulgaris, } \\
\text { segmental }\end{array}$ & $\begin{array}{l}\text { Stable, } \\
\text { active }\end{array}$ & 5 \\
\hline Ma et al. [11] & China & $69 / 44$ & Serum & 3 & $\mathrm{U} / \mathrm{L}$ & $\begin{array}{l}\text { Vulgaris, } \\
\text { segmental }\end{array}$ & - & 5 \\
\hline Wang and $\mathrm{Xu}[12]$ & China & $34 / 30$ & Serum & 1 & $\begin{array}{c}\mathrm{U} / \mathrm{mg} \\
\text { protein }\end{array}$ & - & - & 7 \\
\hline Batçioğlu et al. [13] & Turkey & $37 / 15$ & Plasma & 4 & $\begin{array}{c}\mathrm{U} / \mathrm{mg} \\
\text { protein }\end{array}$ & - & - & 7 \\
\hline Hazneci et al. [14] & Turkey & $23 / 25$ & Plasma & 5 & $\begin{array}{c}\mathrm{U} / \mathrm{mg} \\
\text { protein }\end{array}$ & Vulgaris & Active & 6 \\
\hline Zeng et al. [15] & China & $50 / 50$ & Plasma & - & $\mathrm{U} / \mathrm{L}$ & - & $\begin{array}{l}\text { Stable, } \\
\text { active }\end{array}$ & 5 \\
\hline Liu et al. [16] & China & $60 / 40$ & Plasma & 3 & $\mathrm{U}$ & - & $\begin{array}{l}\text { Stable, } \\
\text { active }\end{array}$ & 7 \\
\hline Karsli et al. [17] & Turkey & $24 / 27$ & Erythrocyte & 1 & $\mathrm{U} / \mathrm{g} \mathrm{Hb}$ & Generalized & - & 7 \\
\hline Ines et al. [18] & Tunisia & $36 / 40$ & Erythrocyte & 6 & $\begin{array}{c}\mathrm{U} / \mathrm{g} \\
\text { protein }\end{array}$ & - & $\begin{array}{l}\text { Stable, } \\
\text { active }\end{array}$ & 5 \\
\hline Hazneci et al. [14] & Turkey & $23 / 25$ & Erythrocyte & 1 & $\mathrm{U} / \mathrm{g} \mathrm{Hb}$ & Vulgaris & Active & 6 \\
\hline Yildirim et al. [19] & Turkey & $24 / 20$ & Erythrocyte & 1 & $\mathrm{U} / \mathrm{g} \mathrm{Hb}$ & Generalized & Stable & 5 \\
\hline Jain et al. [20] & India & $75 / 25$ & Blood & 1 & $\mathrm{U} / \mathrm{L}$ & - & $\begin{array}{l}\text { Stable, } \\
\text { active }\end{array}$ & 5 \\
\hline Jalel and Hamdaoui [21] & Tunisia & $60 / 62$ & Blood & 1 & $\mathrm{U} / \mathrm{L}$ & - & - & 5 \\
\hline Shajil and Begum [22] & India & $124 / 126$ & Blood & 7 & $\begin{array}{c}\mathrm{U} / \mathrm{mg} \\
\text { protein }\end{array}$ & $\begin{array}{c}\text { Segmental, } \\
\text { nonsegmental }\end{array}$ & - & 5 \\
\hline Нa [23] & China & $35 / 31$ & Blood & - & $\mathrm{U}$ & - & Active & 5 \\
\hline Batçioğlu et al. [13] & Turkey & $33 / 15$ & Skin & 4 & $\begin{array}{c}\mathrm{U} / \mathrm{mg} \\
\text { protein }\end{array}$ & - & - & 7 \\
\hline Dammak et al. [24] & Tunisia & $20 / 20$ & Skin & 1 & $\begin{array}{c}\mathrm{U} / \mathrm{mg} \\
\text { protein }\end{array}$ & Vulgaris & $\begin{array}{l}\text { Stable, } \\
\text { active }\end{array}$ & 7 \\
\hline Yildirim et al. [25] & Turkey & $25 / 25$ & Skin & 1 & $\begin{array}{c}\mathrm{U} / \mathrm{mg} \\
\text { protein }\end{array}$ & Generalized & Stable & 5 \\
\hline Passi et al. [26] & Italy & $15 / 15$ & Skin & 1 & $\begin{array}{c}\mathrm{U} / \mathrm{mg} \\
\text { protein }\end{array}$ & - & Active & 7 \\
\hline Zeng et al. [15] & China & $50 / 50$ & Blister fluid & - & $\mathrm{U} / \mathrm{L}$ & - & $\begin{array}{l}\text { Stable, } \\
\text { active }\end{array}$ & 5 \\
\hline Li et al. [27] & China & $24 / 10$ & Blister fluid & 3 & $\mathrm{U}$ & - & $\begin{array}{l}\text { Stable, } \\
\text { active }\end{array}$ & 7 \\
\hline Xu et al. [28] & China & 19 (self control) & Blister fluid & 3 & $\mathrm{U}$ & - & Stable & 7 \\
\hline
\end{tabular}

Note. NOS, Newcastle-Ottawa Scale; 1: Paglia and Valentine's method (1967); 2: flameless atomic absorption (graphite furnace) method; 3: DTNB colorimetry; 4: Lawrence and Burk's method (1976); 5: Najwa's method (1990); 6: Paglia's method (2002); 7: Beutler's method (1989). 
TABLE 2: The level of glutathione peroxidase of vitiligo patients stratified by sample sources and races (mean \pm SD).

\begin{tabular}{|c|c|c|c|c|c|}
\hline Study & Race & Unit & Case & Control & Source \\
\hline Zedan et al. [3] & Caucasian & $\mathrm{U} / \mathrm{L}$ & $0.29 \pm 0.14$ & $0.47 \pm 0.13$ & Serum \\
\hline Barikbin et al. [7] & Caucasian & $\mathrm{U} / \mathrm{L}$ & $191.300 \pm 14.95$ & $183.288 \pm 13.42$ & Serum \\
\hline Ozturk et al. [8] & Caucasian & $\mathrm{U} / \mathrm{mg}$ protein & $0.550 \pm 0.077$ & $0.439 \pm 0.075$ & Serum \\
\hline Zhao et al. [9] & Asian & - & $292.21 \pm 127.42$ & $280.88 \pm 47.25$ & Serum \\
\hline Chen et al. [10] & Asian & $\mathrm{U} / \mathrm{L}$ & $140030 \pm 15260$ & $216440 \pm 8610$ & Serum \\
\hline Ma et al. [11] & Asian & $\mathrm{U} / \mathrm{L}$ & $102.08 \pm 19.32$ & $154.76 \pm 27.06$ & Serum \\
\hline Wang and $\mathrm{Xu}[12]$ & Asian & $\mathrm{U} / \mathrm{mg}$ protein & $0.000981 \pm 0.000257$ & $0.001722 \pm 0.000602$ & Serum \\
\hline Batçioğlu et al. [13] & Caucasian & $\mathrm{U} / \mathrm{mg}$ protein & $381.57 \pm 12.67$ & $346.13 \pm 21.90$ & Plasma \\
\hline Hazneci et al. [14] & Caucasian & $\mathrm{U} / \mathrm{mg}$ protein & $0.205 \pm 0.192$ & $0.171 \pm 0.012$ & Plasma \\
\hline Zeng et al. [15] & Asian & $\mathrm{U} / \mathrm{L}$ & $98.44 \pm 14.23$ & $114.33 \pm 10.41$ & Plasma \\
\hline Liu et al. [16] & Asian & $\mathrm{U}$ & $96.40 \pm 15.78$ & $115.14 \pm 12.20$ & Plasma \\
\hline Karsli et al. [17] & Caucasian & $\mathrm{U} / \mathrm{g} \mathrm{Hb}$ & $13.71 \pm 3.85$ & $18.87 \pm 4.42$ & Erythrocyte \\
\hline Ines et al. [18] & Caucasian & $\mathrm{U} / \mathrm{g}$ protein & $1160 \pm 440$ & $1890 \pm 820$ & Erythrocyte \\
\hline Hazneci et al. [14] & Caucasian & $\mathrm{U} / \mathrm{g} \mathrm{Hb}$ & $97836.86 \pm 17947.61$ & $90257.83 \pm 15776.65$ & Erythrocyte \\
\hline Yildirim et al. [19] & Caucasian & $\mathrm{U} / \mathrm{g} \mathrm{Hb}$ & $31.34 \pm 14.3$ & $101.57 \pm 48.4$ & Erythrocyte \\
\hline Jain et al. [20] & Caucasian & $\mathrm{U} / \mathrm{L}$ & $4004 \pm 482.34$ & $3945 \pm 552$ & Blood \\
\hline Jalel and Hamdaoui [21] & Caucasian & $\mathrm{U} / \mathrm{L}$ & $42 \pm 23.24$ & $49 \pm 14.17$ & Blood \\
\hline Shajil and Begum [22] & Caucasian & $\mathrm{U} / \mathrm{mg}$ protein & $944.55 \pm 160.92$ & $1036.8 \pm 269.6$ & Blood \\
\hline Ha [23] & Asian & $\mathrm{U}$ & $128.18 \pm 18.35$ & $206.14 \pm 21.50$ & Blood \\
\hline Batçioğlu et al. [13] & Caucasian & $\mathrm{U} / \mathrm{mg}$ protein & $170.98 \pm 12.35$ & $121.91 \pm 16.03$ & Skin \\
\hline Dammak et al. [24] & Caucasian & $\mathrm{U} / \mathrm{mg}$ protein & $2.73 \pm 0.81$ & $1.78 \pm 0.43$ & Skin \\
\hline Yildirim et al. [25] & Caucasian & $\mathrm{U} / \mathrm{mg}$ protein & $3.72 \pm 2.09$ & $1.58 \pm 0.48$ & Skin \\
\hline Passi et al. [26] & Caucasian & $\mathrm{U} / \mathrm{mg}$ protein & $0.43 \pm 0.10$ & $0.47 \pm 0.08$ & Skin \\
\hline Zeng et al. [15] & Asian & $\mathrm{U} / \mathrm{L}$ & $86.53 \pm 7.83$ & $98.26 \pm 8.87$ & Blister fluid \\
\hline Li et al. [27] & Asian & $\mathrm{U}$ & $148.73 \pm 51.91$ & $115.01 \pm 29.57$ & Blister fluid \\
\hline $\mathrm{Xu}$ et al. [28] & Asian & $\mathrm{U}$ & $90.67 \pm 63.07$ & $96.76 \pm 63.51$ & Blister fluid \\
\hline
\end{tabular}

TABLE 3: The level of glutathione peroxidase of vitiligo patients stratified by stage and healthy controls (mean \pm SD).

\begin{tabular}{|c|c|c|c|c|c|}
\hline \multirow{2}{*}{ Study } & \multirow{2}{*}{ Unit } & \multicolumn{2}{|c|}{ Case } & \multirow{2}{*}{ Control } & \multirow{2}{*}{ Source } \\
\hline & & Stable & Active & & \\
\hline Zedan et al. [3] & $\mathrm{U} / \mathrm{L}$ & $0.27 \pm 0.17(n=9)$ & $0.29 \pm 0.14(n=48)$ & $0.47 \pm 0.13(n=30)$ & Serum \\
\hline Zhao et al. [9] & - & $346.93 \pm 156.84(n=14)$ & $262.75 \pm 103.54(n=26)$ & $280.88 \pm 47.25(n=10)$ & Serum \\
\hline Chen et al. [10] & $\mathrm{U} / \mathrm{L}$ & $139120 \pm 11760(n=12)$ & $140940 \pm 13210(n=120)$ & $216440 \pm 8610(n=50)$ & Serum \\
\hline Zeng et al. [15] & $\mathrm{U} / \mathrm{L}$ & $104.39 \pm 11.49(n=30)$ & $88.74 \pm 18.23(n=20)$ & $114.33 \pm 10.41(n=50)$ & Plasma \\
\hline Liu et al. [16] & $\mathrm{U}$ & $103.08 \pm 14.95(n=30)$ & $89.72 \pm 13.80(n=30)$ & $115.14 \pm 12.20(n=40)$ & Plasma \\
\hline Ines et al. [18] & $\mathrm{U} / g$ protein & $1250 \pm 470(n=18)$ & $1060 \pm 380(n=18)$ & $1890 \pm 820(n=40)$ & Erythrocyte \\
\hline Jain et al. [20] & $\mathrm{U} / \mathrm{L}$ & $3990 \pm 459(n=25)$ & $4011 \pm 498(n=50)$ & $3945 \pm 552(n=25)$ & Blood \\
\hline $\mathrm{Ha}[23]$ & $\mathrm{U} / \mathrm{mg}$ protein & $2.48 \pm 0.81(n=10)$ & $2.98 \pm 0.77(n=10)$ & $1.78 \pm 0.43(n=20)$ & Skin \\
\hline Zeng et al. [15] & $\mathrm{U} / \mathrm{L}$ & $95.33 \pm 9.37(n=30)$ & $80.69 \pm 10.38(n=20)$ & $98.26 \pm 8.87(n=50)$ & Blister fluid \\
\hline Passi et al. [26] & $\mathrm{U}$ & $92.32 \pm 23.67(n=9)$ & $182.58 \pm 73.67(n=15)$ & $115.01 \pm 29.57(n=10)$ & Blister fluid \\
\hline
\end{tabular}

was no obvious evidence of publication bias $(t=0.32, p=$ $0.754)$.

\section{Discussion}

Oxidative stress inducing vitiligo is based on the fact that some intermediates such as 3,4-dihydroxyphenylalanine (dopa), dopachrome, and 5,6-dihydroxyindole are created during melanin biosynthesis [29]. The final result of these changes results in the continuous increase of hydrogen peroxide $\left(\mathrm{H}_{2} \mathrm{O}_{2}\right)$, which restrains the antioxidative enzyme activity leading to the destruction of melanocytes [30]. Therefore, antioxidants are important to nullify the harmful radical-mediated reactions. GPx is a group of antioxidative markers against free radicals by detoxification and has been considered to be involved in the pathogenesis of many skin diseases [31-33]. Our meta-analysis investigated whether GPx is involved in the development of vitiligo. The results of 


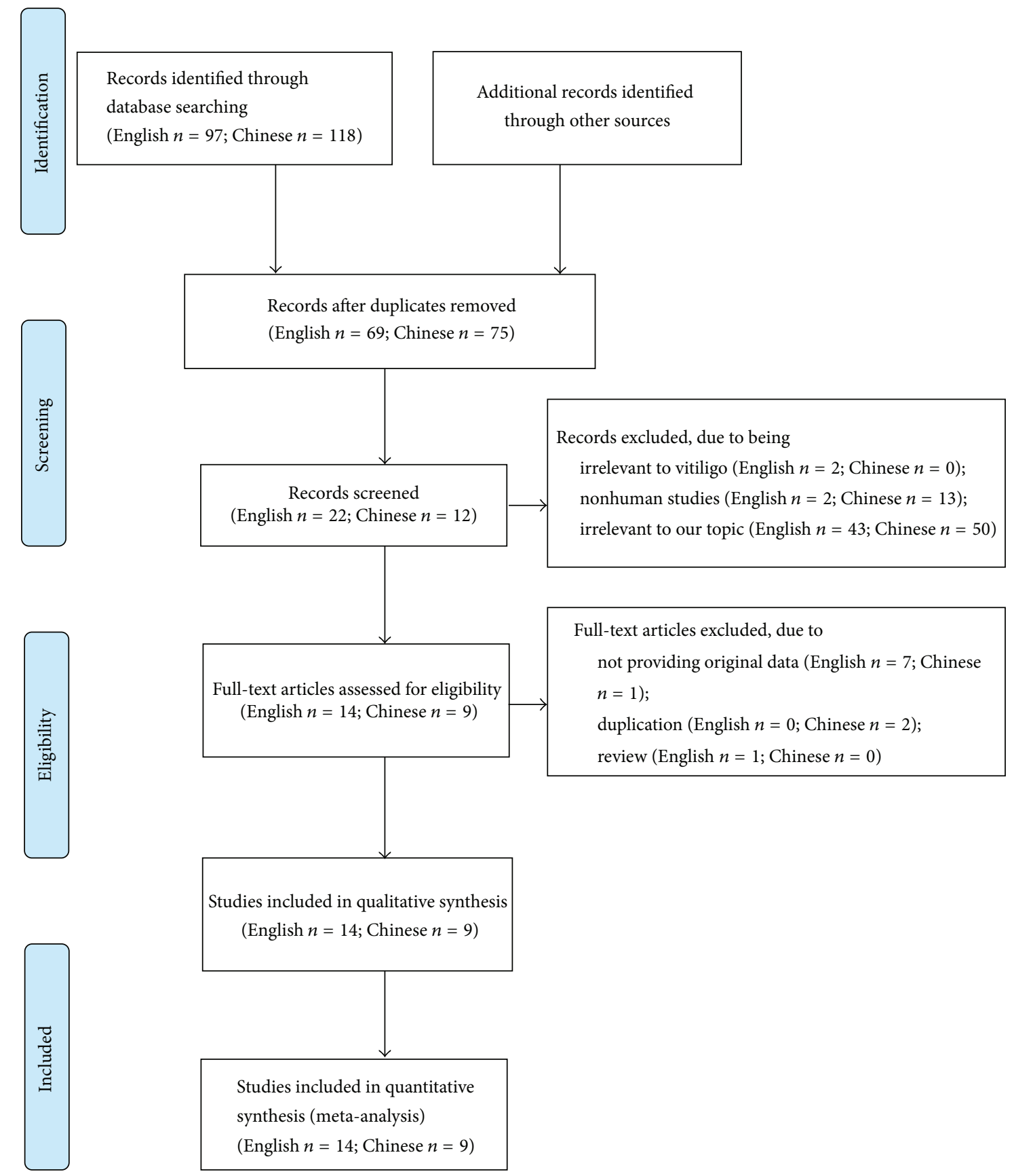

FIGURE 1: Flow diagram of screened and included studies.

TABLE 4: The level of glutathione peroxidase of segmental vitiligo patients, nonsegmental vitiligo patients, and healthy controls (mean \pm SD).

\begin{tabular}{|c|c|c|c|c|c|}
\hline \multirow{2}{*}{ Study } & \multirow{2}{*}{ Unit } & \multicolumn{2}{|c|}{ Case } & \multirow{2}{*}{ Control } & \multirow{2}{*}{ Source } \\
\hline & & Segmental & Nonsegmental & & \\
\hline Chen et al. [10] & $\mathrm{U} / \mathrm{L}$ & $141500 \pm 9210(n=8)$ & $138560 \pm 14620(n=124)$ & $216440 \pm 8610(n=50)$ & Serum \\
\hline Ma et al. [11] & $\mathrm{U} / \mathrm{L}$ & $98.33 \pm 20.34(n=23)$ & $103.95 \pm 18.73(n=46)$ & $154.76 \pm 27.06(n=44)$ & Serum \\
\hline Shajil and Begum [22] & $\mathrm{U} / \mathrm{mg}$ protein & $916.8 \pm 183.3(n=30)$ & $953.4 \pm 151.9(n=94)$ & $1036.8 \pm 269.6(n=126)$ & Blood \\
\hline
\end{tabular}




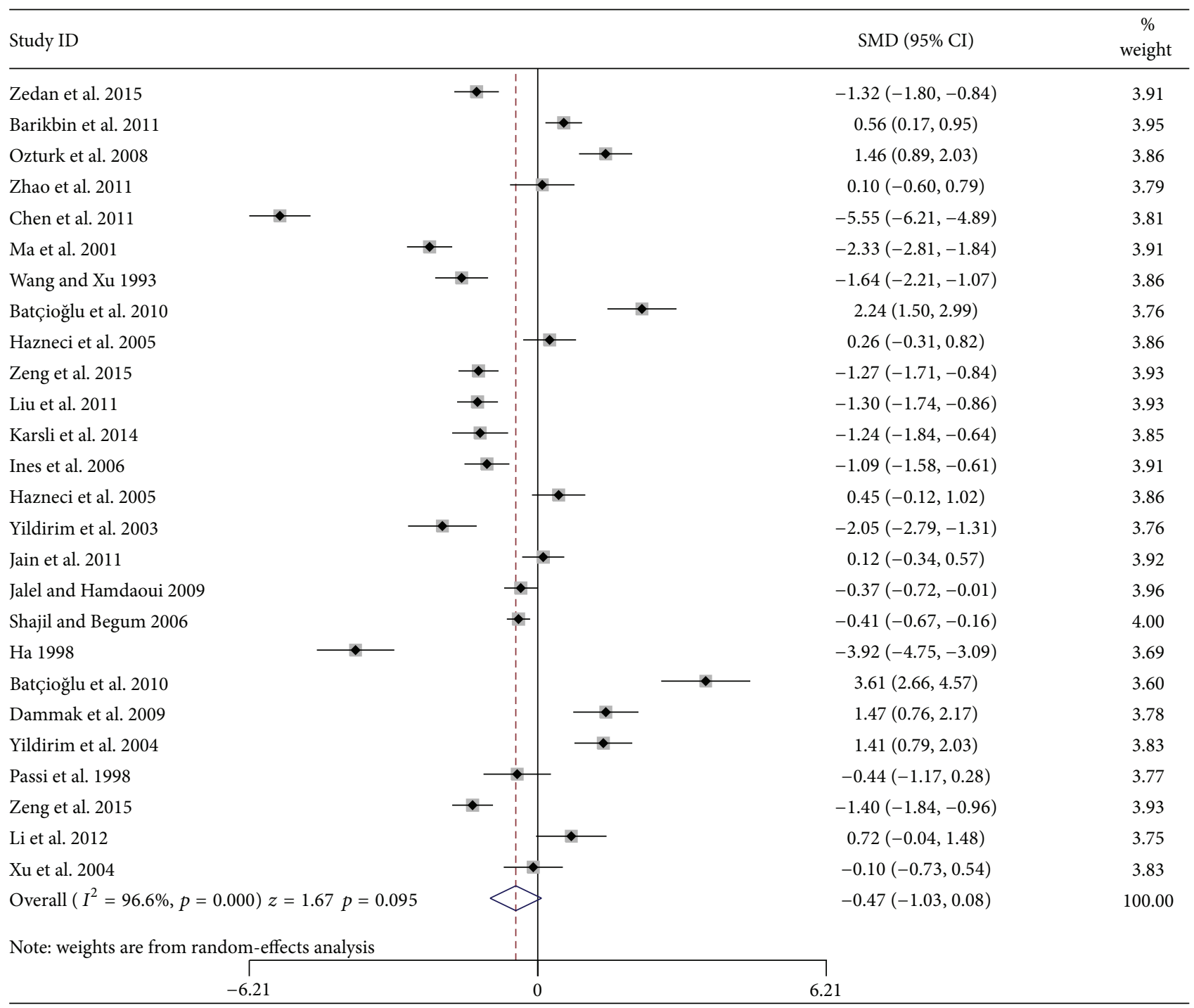

FIGURE 2: Forest plots of studies in glutathione peroxidase levels for subjects with vitiligo patients versus healthy controls.

TABLE 5: Metaregression analyses of potential source of heterogeneity.

\begin{tabular}{|c|c|c|c|c|c|c|}
\hline \multirow{2}{*}{ Heterogeneity factors } & \multirow{2}{*}{ Coefficient } & \multirow{2}{*}{ Std. Err. } & \multirow{2}{*}{$z$} & \multirow{2}{*}{$p>|z|$} & \multicolumn{2}{|c|}{$95 \% \mathrm{CI}$} \\
\hline & & & & & LL & UL \\
\hline \multicolumn{7}{|l|}{ Race } \\
\hline Univariate & -1.940708 & .6522389 & -2.98 & 0.003 & -3.219072 & -.6623431 \\
\hline Multivariate & -1.811697 & .6340008 & -2.86 & 0.004 & -3.054315 & -.569078 \\
\hline \multicolumn{7}{|l|}{ Country } \\
\hline Univariate & -.158666 & .2952626 & -0.54 & 0.591 & -.73737 & .420038 \\
\hline Multivariate & -.3211 & .27018 & -1.19 & 0.235 & -.8506431 & .2084431 \\
\hline \multicolumn{7}{|l|}{ Sample Source } \\
\hline Univariate & .3021601 & .2049701 & 1.47 & 0.140 & -.099574 & .7038942 \\
\hline Multivariate & .370906 & .1947404 & 1.90 & 0.057 & -.0107781 & .7525901 \\
\hline
\end{tabular}

Note. Std. Err., standard error; 95\% CI, 95\% confidence interval; UL, upper limit; LL, lower limit. 


\begin{tabular}{|c|c|c|c|c|}
\hline Study ID & & & SMD $(95 \% \mathrm{CI})$ & $\begin{array}{c}\% \\
\text { weight }\end{array}$ \\
\hline \multicolumn{5}{|l|}{ Serum } \\
\hline Zedan et al. 2015 & $\multimap$ & & $-1.32(-1.80,-0.84)$ & 14.36 \\
\hline Barikbin et al. 2011 & & $\rightarrow$ & $0.56(0.17,0.95)$ & 14.42 \\
\hline Ozturk et al. 2008 & & $\longrightarrow$ & $1.46(0.89,2.03)$ & 14.27 \\
\hline Zhao et al. 2011 & & 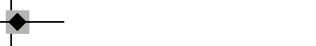 & $0.10(-0.60,0.79)$ & 14.14 \\
\hline Chen et al. 2011 & & & $-5.55(-6.21,-4.89)$ & 14.18 \\
\hline Ma et al. 2001 & $\neg$ & & $-2.33(-2.81,-1.84)$ & 14.35 \\
\hline Wang and Xu 1993 & $\longrightarrow$ & & $-1.64(-2.21,-1.07)$ & 14.28 \\
\hline Subtotal $\left(I^{2}=98.3 \%, p=0.000\right) z=1.57 p=0.117$ & $\sim-$ & $p$ & $-1.24(-2.79,0.31)$ & 100.00 \\
\hline \multicolumn{5}{|l|}{ Plasma } \\
\hline Batçioğlu et al. 2010 & & $\longrightarrow$ & $2.24(1.50,2.99)$ & 24.23 \\
\hline Hazneci et al. 2005 & & 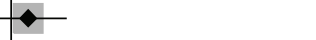 & $0.26(-0.31,0.82)$ & 24.96 \\
\hline Zeng et al. 2015 & $\neg$ & & $-1.27(-1.71,-0.84)$ & 25.42 \\
\hline Liu et al. 2011 & $\rightarrow$ & & $-1.30(-1.74,-0.86)$ & 25.39 \\
\hline Subtotal $\left(I^{2}=96.4 \%, p=0.000\right) z=0.07 p=0.948$ & & P & $-0.05(-1.43,1.34)$ & 100.00 \\
\hline \multicolumn{5}{|l|}{ Erythrocyte } \\
\hline Karsli et al. 2014 & $\multimap$ & & $-1.24(-1.84,-0.64)$ & 25.01 \\
\hline Ines et al. 2006 & $\multimap$ & & $-1.09(-1.58,-0.61)$ & 25.90 \\
\hline Hazneci et al. 2005 & & 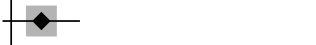 & $0.45(-0.12,1.02)$ & 25.24 \\
\hline Yildirim et al. 2003 & $\longrightarrow$ & & $-2.05(-2.79,-1.31)$ & 23.85 \\
\hline Subtotal $\left(I^{2}=90.8 \%, p=0.000\right) z=1.96 p=0.050$ & & & $-0.97(-1.94,0.00)$ & 100.00 \\
\hline \multicolumn{5}{|l|}{ Blood } \\
\hline Jain et al. 2011 & & 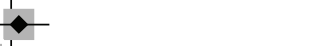 & $0.12(-0.34,0.57)$ & 25.34 \\
\hline Jalel and Hamdaoui 2009 & & & $-0.37(-0.72,-0.01)$ & 25.84 \\
\hline Shajil and Begum 2006 & & & $-0.41(-0.67,-0.16)$ & 26.29 \\
\hline На 1998 & & & $-3.92(-4.75,-3.09)$ & 22.53 \\
\hline Subtotal $\left(I^{2}=95.9 \%, p=0.000\right) z=2.07 p=0.038$ & & & $-1.06(-2.05,-0.06)$ & 100.00 \\
\hline \multicolumn{5}{|l|}{ Skin } \\
\hline Batçioğlu et al. 2010 & & $\longrightarrow$ & $3.61(2.66,4.57)$ & 23.99 \\
\hline Dammak et al. 2009 & & $\longrightarrow$ & $1.47(0.76,2.17)$ & 25.26 \\
\hline Yildirim et al. 2004 & & $\multimap$ & $1.41(0.79,2.03)$ & 25.60 \\
\hline Passi et al. 1998 & & + & $-0.44(-1.17,0.28)$ & 25.16 \\
\hline Subtotal $\left(I^{2}=93.3 \%, p=0.000\right) z=2.05 p=0.041$ & & $\longrightarrow$ & $1.49(0.06,2.91)$ & 100.00 \\
\hline \multicolumn{5}{|l|}{ Blister fluid } \\
\hline Zeng et al. 2015 & 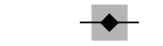 & & $-1.40(-1.84,-0.96)$ & 34.72 \\
\hline Li et al. 2012 & & $\longrightarrow$ & $0.72(-0.04,1.48)$ & 32.07 \\
\hline Xu et al. 2004 & & 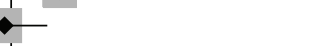 & $-0.10(-0.73,0.54)$ & 33.20 \\
\hline Subtotal $\left(I^{2}=92.5 \%, p=0.000\right) z=0.44 p=0.657$ & & $F$ & $-0.29(-1.56,0.98)$ & 100.00 \\
\hline \multicolumn{5}{|l|}{ Note: weights are from random-effects analysis } \\
\hline $\begin{array}{c}\mid \\
-6.21\end{array}$ & & $\begin{array}{l}1 \\
0\end{array}$ & 6.21 & \\
\hline
\end{tabular}

(a)

Figure 3: Continued. 


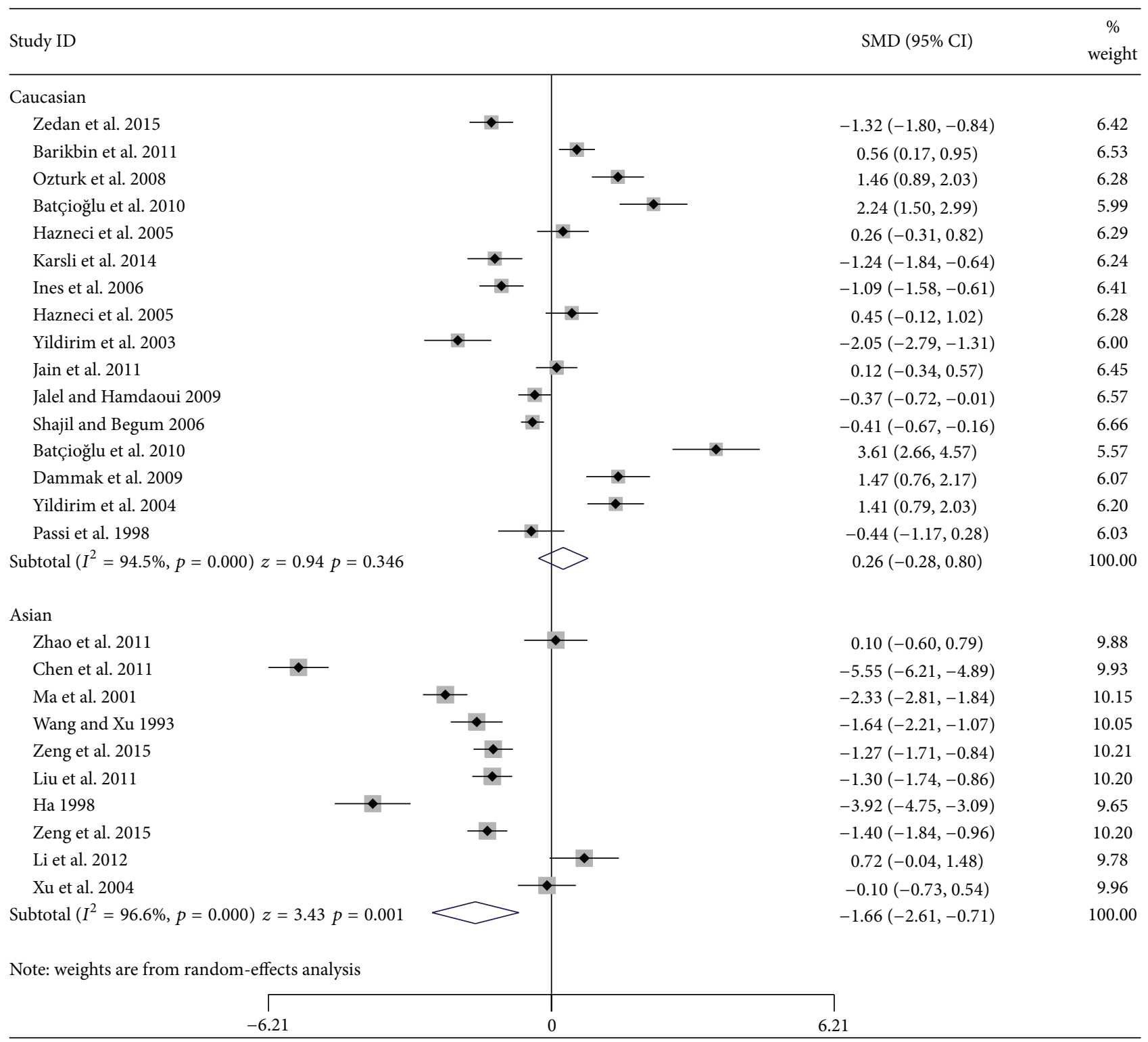

(b)

FIGURE 3: Subgroup analyses of studies in glutathione peroxidase levels for subjects with vitiligo versus healthy controls stratified by (a) sample sources and (b) races.

included articles involving 26 comparisons on the relationship of GPx and vitiligo were controversial; that is, respective $50 \%, 31 \%$, and $19 \%$ comparisons showed lower, equal, and higher levels in vitiligo samples. The difference may relate to the variations in the population race, disease type, activity, duration, sample sources, or detection method.

Till now, no meta-analysis has reported the association between the GPx level and vitiligo. The pooled meta-analysis results of all the comparisons indicated that the GPx levels in vitiligo patients were similar to healthy controls. As statistical heterogeneity existed among studies, we did further subgroup analysis. The results indicated a significant relationship between low GPx level and vitiligo incidence.
Our subgroup analysis showed that Asian vitiligo patients showed lower levels of GPx than the controls, but no difference was shown between Caucasian populations and healthy controls. The metaregression results, which showed that race could be the major source of heterogeneity of pooled meta-analysis, supported the above subgroup analysis results. The majority of previous studies have used serum or plasma to measure oxidant or antioxidant levels. In the present meta-analysis, whatever stable vitiligo patients or active vitiligo patients had lower serum/plasma levels of GPx than the controls. The patients with segmental type also had decreased GPx levels comparing to healthy controls. These results suggested that low GPx level may contribute 


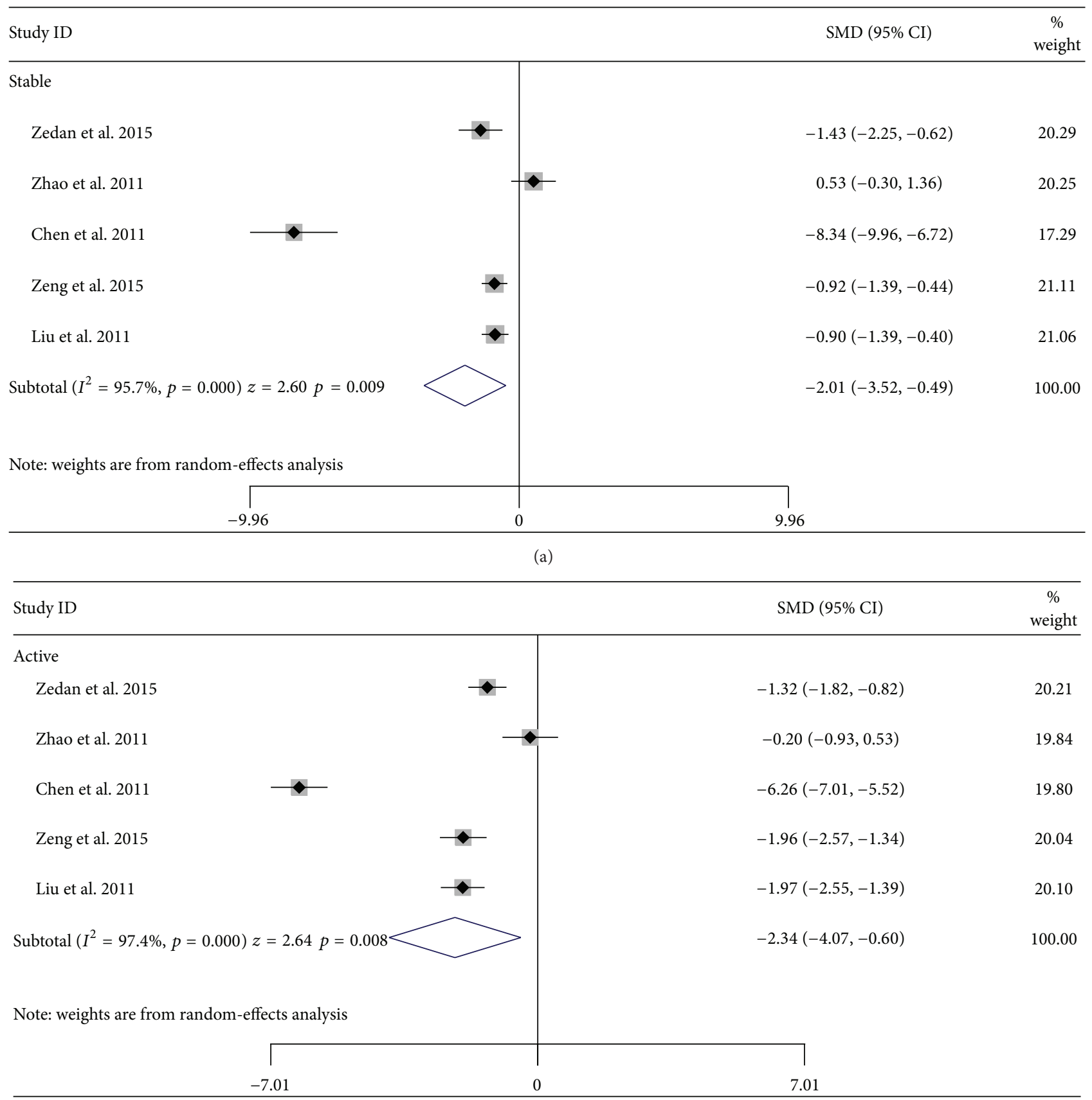

(b)

FIGURE 4: Subgroup analyses of studies in glutathione peroxidase levels in serum/plasma stratified by vitiligo stage. (a) Vitiligo at stable stage versus healthy controls and (b) vitiligo at active stage versus healthy controls.

to the pathogenesis of vitiligo in Asian population, unlike Caucasian population. The low level in serum/plasma was associated with vitiligo incidence, at whatever active stage or stable stage, especially in segmental vitiligo. Oxidative stress induced accumulation of toxic-free radicals may have a pathophysiologic role in the initiation and progression of vitiligo [2]. Reactive oxygen species (ROS) are scavenged by antioxidant defence mechanisms. Depletion of the endogenous antioxidants including GPx can overwhelm antioxidant defence mechanisms, resulting in oxidative stress medicated vitiligo. Besides, allelic variants in GPx gene may be associated with low levels of GPx activity [34, 35]. One previous study indicated that GPx polymorphism may contribute to the reduced GPx activity and the prevalence of vitiligo in Gujarat population [36].

In conclusion, this meta-analysis showed a significant association between low GPx level and vitiligo for Asian population or segmental patients. The low level in serum/plasma 


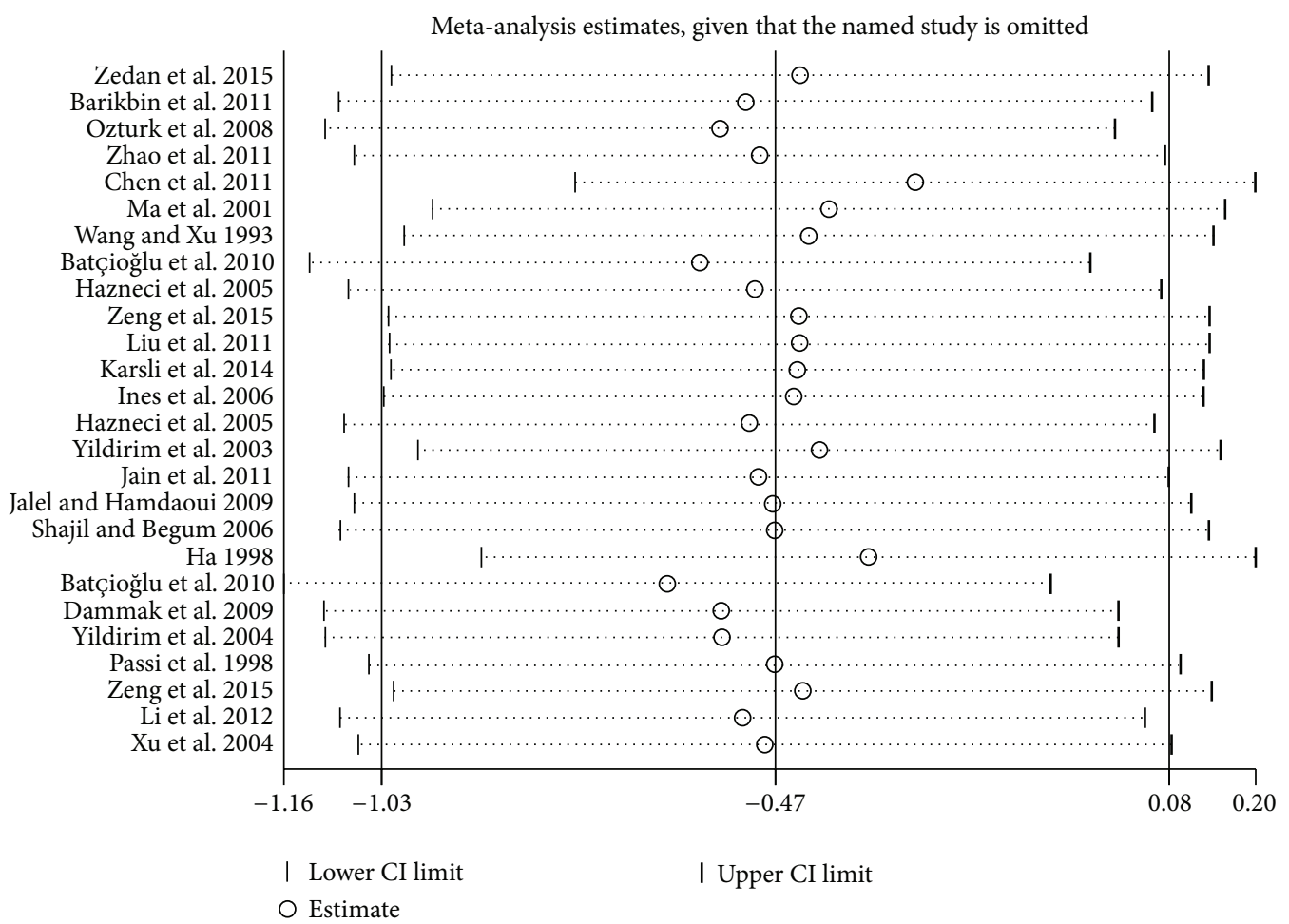

FIGURE 5: Forest plots for the sensitive analysis.

was associated with vitiligo incidence, at whatever active or stable stage. Nonetheless, the conclusion could not be completely confirmed as there are some limitations. The limited number, small sample sizes of studies, and methodological diversities may weaken the statistical power. More largesample studies of higher quality should be done to verify the conclusions.

\section{Competing Interests}

The authors have no commercial associations that might create competing interests in connection with the submitted paper.

\section{Acknowledgments}

This work has been supported by National Natural Science Fund (code: 81301388) and Liaoning Province Education Bureau Project (code: LZ2015077).

\section{References}

[1] R. M. Halder and J. L. Chappell, "Vitiligo update," Seminars in Cutaneous Medicine and Surgery, vol. 28, no. 2, pp. 86-92, 2009.

[2] K. U. Schallreuter, J. M. Wood, and J. Berger, "Low catalase levels in the epidermis of patients with vitiligo," The Journal of Investigative Dermatology, vol. 97, no. 6, pp. 1081-1085, 1991.

[3] H. Zedan, A. A. L. Abdel-Motaleb, N. M. A. A. Kassem, H. A. H. A. Hafeez, and M. R. E. A. Hussein, "Low glutathione peroxidase activity levels in patients with vitiligo," Journal of Cutaneous Medicine and Surgery, vol. 19, no. 2, pp. 144-148, 2015.
[4] A. Stang, "Critical evaluation of the Newcastle-Ottawa scale for the assessment of the quality of nonrandomized studies in metaanalyses," European Journal of Epidemiology, vol. 25, no. 9, pp. 603-605, 2010.

[5] J. L. Peters, A. J. Sutton, D. R. Jones, K. R. Abrams, and L. Rushton, "Comparison of two methods to detect publication bias in meta-analysis," The Journal of the American Medical Association, vol. 295, no. 6, pp. 676-680, 2006.

[6] E. Zintzaras and J. P. A. Ioannidis, "HEGESMA: genome search meta-analysis and heterogeneity testing," Bioinformatics, vol. 21, no. 18, pp. 3672-3673, 2005.

[7] B. Barikbin, S. Kavand, M. Yousefi, M. Hedayati, and M. Saeedi, "No differences in serum selenium levels and blood glutathione peroxidase activities in patients with vitiligo compared with healthy control subjects," Journal of the American Academy of Dermatology, vol. 64, no. 2, pp. 444-445, 2011.

[8] I. C. Ozturk, K. Batcioglu, F. Karatas, E. Hazneci, and M. Genc, "Comparison of plasma malondialdehyde, glutathione, glutathione peroxidase, hydroxyproline and selenium levels in patients with vitiligo and healthy controls," Indian Journal of Dermatology, vol. 53, no. 3, pp. 106-110, 2008.

[9] J. Zhao, W. Li, and S. Y. Li, “The detection of hydrogen peroxide, catalase and glutathion peroxide in serum of vitiligo," Chinese Journal of Dermatology and Venereology, vol. 25, no. 10, pp. 739740, 2011.

[10] J. P. Chen, C. Y. Zhong, and Y. P. Kang, "Serum antioxidative enzyme and lipid peroxide in vitiligo patients," Zhejiang Journal of Laboratory Medicine, vol. 9, no. 3, pp. 24-25, 2011.

[11] H. Y. Ma, X. N. Ni, and G. S. Xue, "Detection of glutathione and glutathione peroxidase in the sera of patients with vitiligo," Journal of Clinical Dermatology, vol. 30, no. 1, pp. 22-23, 2001. 
[12] F. Wang and H. Q. Xu, "Some enzymes and microelements change research in sera and tissue of vitiligo patients," Chinese Journal of Dermatology and Venereology, vol. 7, no. 3, pp. 142143, 1993.

[13] K. Batçioğlu, E. Haznec1, Ç. Öztürk, A. B. Karabulut, and N. Karadağ, "The skin and plasma antioxidant enzyme activities in patients with vitiligo," Trakya Üniversitesi Tıp Fakültesi Dergisi, vol. 27, no. 4, pp. 354-357, 2010.

[14] E. Hazneci, A. B. Karabulut, Ç. Öztürk et al., "A comparative study of superoxide dismutase, catalase, and glutathione peroxidase activities and nitrate levels in vitiligo patients," International Journal of Dermatology, vol. 44, no. 8, pp. 636640, 2005.

[15] Z. P. Zeng, B. Z. Lin, and T. H. Ge, "A comparative study of oxidation and antioxidant capacity of skin and serum in patients with vitiligo," Journal of Dermatology and Venereology, vol. 37, no. 3, pp. 140-142, 2015.

[16] Z. J. Liu, J. Liu, and X. H. Tang, "Related parameters of oxidation and antioxidation in sera of vitiligo patients," The Chinese Journal of Dermatovenereology, vol. 25, no. 4, pp. 255-257, 2011.

[17] N. Karsli, C. Akcali, O. Ozgoztasi, N. Kirtak, and S. Inaloz, "Role of oxidative stress in the pathogenesis of vitiligo with special emphasis on the antioxidant action of narrowband ultraviolet B phototherapy," The Journal of International Medical Research, vol. 42, no. 3, pp. 799-805, 2014.

[18] D. Ines, B. Sonia, B. M. Riadh et al., "A comparative study of oxidant-antioxidant status in stable and active vitiligo patients," Archives of Dermatological Research, vol. 298, no. 4, pp. 147-152, 2006.

[19] M. Yildirim, V. Baysal, H. S. Inaloz, D. Kesici, and N. Delibas, "The role of oxidants and antioxidants in generalized vitiligo," The Journal of Dermatology, vol. 30, no. 2, pp. 104-108, 2003.

[20] A. Jain, J. Mal, V. Mehndiratta, R. Chander, and S. K. Patra, "Study of oxidative stress in vitiligo," Indian Journal of Clinical Biochemistry, vol. 26, no. 1, pp. 78-81, 2011.

[21] A. Jalel and M. H. Hamdaoui, "Study of total antioxidant status and glutathione peroxidase activity in Tunisian vitiligo patients," Indian Journal of Dermatology, vol. 54, no. 1, pp. 13$16,2009$.

[22] E. M. Shajil and R. Begum, "Antioxidant status of segmental and non-segmental vitiligo," Pigment Cell Research, vol. 19, no. 2, pp. 179-180, 2006.

[23] M. L. Ha, "Peripheral blood free radical level changes of vitiligo patients," Shanghai Journal of Immunology, vol. 18, no. 1, pp. 4647, 1998.

[24] I. Dammak, S. Boudaya, F. Ben Abdallah, H. Turki, H. Attia, and B. Hentati, "Antioxidant enzymes and lipid peroxidation at the tissue level in patients with stable and active vitiligo," International Journal of Dermatology, vol. 48, no. 5, pp. 476-480, 2009.

[25] M. Yildirim, V. Baysal, H. S. Inaloz, and M. Can, "The role of oxidants and antioxidants in generalized vitiligo at tissue level," Journal of the European Academy of Dermatology and Venereology, vol. 18, no. 6, pp. 683-686, 2004.

[26] S. Passi, M. Grandinetti, F. Maggio, A. Stancato, and C. De Luca, "Epidermal oxidative stress in vitiligo," Pigment Cell Research, vol. 11, no. 2, pp. 81-85, 1998.

[27] W. Li, S. Y. Li, and J. Q. Lu, "A comparative study on levels of oxidant-antioxidant in the tissue fluid from skin of vitiligo," Chinese Journal of Dermatology and Venereology, vol. 26, no. 4, pp. 303-305, 2012.
[28] Y. Xu, S. X. Yan, and Y. Hu, "Comparison of level of anti-oxidant enzyme and lipid peroxidation in the blister fluid from vitiligo lesions with that of normal skin," Laboratory Medicine, vol. 19, no. 4, pp. 321-323, 2004.

[29] S. K. Hann and W. Chun, "Autocytotoxic hypothesis for the destruction of melanocytes as the cause of vitiligo," in Vitiligo: A Monograph on the Basic and Clinical Science, S. K. Hann and J. Nordlund, Eds., pp. 137-141, Blackwell Science, Oxford, UK, 2001.

[30] D. Agrawal, E. M. Shajil, Y. S. Marfatia, and R. Begum, "Study on the antioxidant status of vitiligo patients of different age groups in Baroda," Pigment Cell Research, vol. 17, no. 3, pp. 289-294, 2004.

[31] M. H. Javanbakht, M. Djalali, M. Daneshpazhooh et al., "Evaluation of antioxidant enzyme activity and antioxidant capacity in patients with newly diagnosed pemphigus vulgaris," Clinical and Experimental Dermatology, vol. 40, no. 3, pp. 313-317, 2015.

[32] M. Naziroglu, M. Sahin, B. Cig, M. Aykur, I. Erturan, and Y. Ugan, "Hypericum perforatum modulates apoptosis and calcium mobilization through voltage-gated and TRPM2 calcium channels in neutrophil of patients with Behcet's disease," The Journal of Membrane Biology, vol. 247, no. 3, pp. 253-262, 2014.

[33] H. M. Dutchak, "The protein oxidation and antioxidant defence mechanisms in children with atopic dermatitis," Likars'ka Sprava/Ministerstvo Okhorony Zdorov'ia Ukrainy, no. 5, pp. 8994, 2013.

[34] Y. J. Hu and A. M. Diamond, "Role of glutathione peroxidase 1 in breast cancer: loss of heterozygosity and allelic differences in the response to selenium," Cancer Research, vol. 63, no. 12, pp. 3347-3351, 2003.

[35] D. Ratnasinghe, J. A. Tangrea, M. R. Andersen et al., "Glutathione peroxidase codon 198 polymorphism variant increases lung cancer risk," Cancer Research, vol. 60, no. 22, pp. 6381-6383, 2000.

[36] S. Em, N. C. Laddha, S. C. hatterjee et al., "Association of catalase T/C exon 9 and glutathione peroxidase codon 200 polymorphisms in relation to their activities and oxidative stress with vitiligo susceptibility in Gujarat population," Pigment Cell Research/Sponsored by the European Society for Pigment Cell Research and the International Pigment Cell Society, vol. 20, no. 5, pp. 405-407, 2007. 


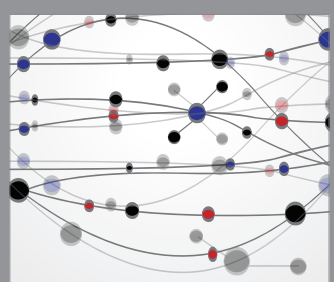

The Scientific World Journal
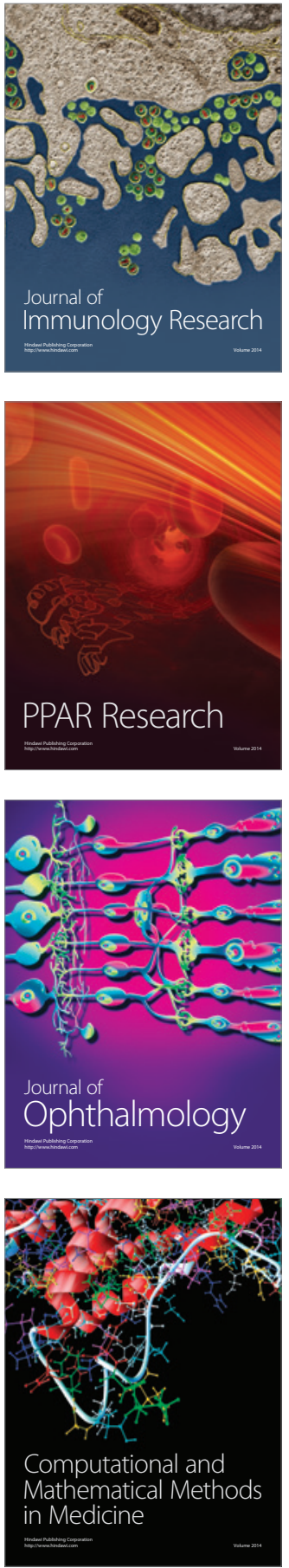

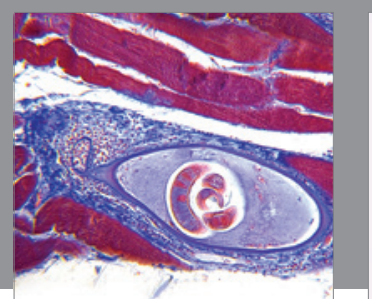

Gastroenterology Research and Practice

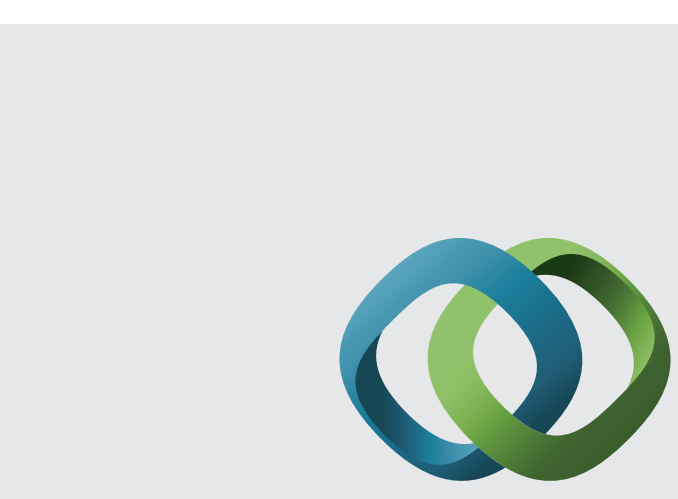

\section{Hindawi}

Submit your manuscripts at

http://www.hindawi.com
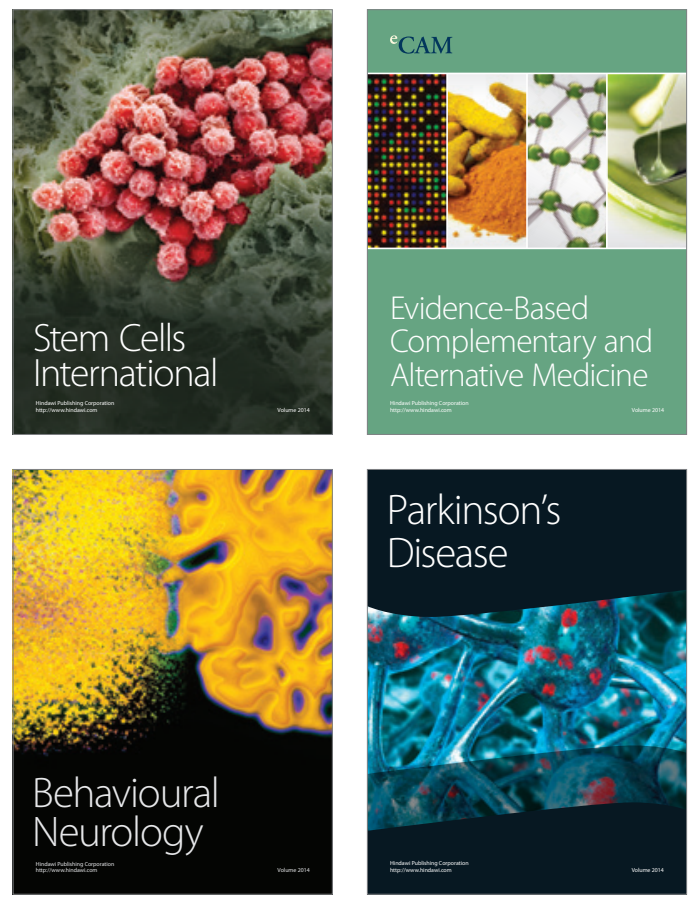
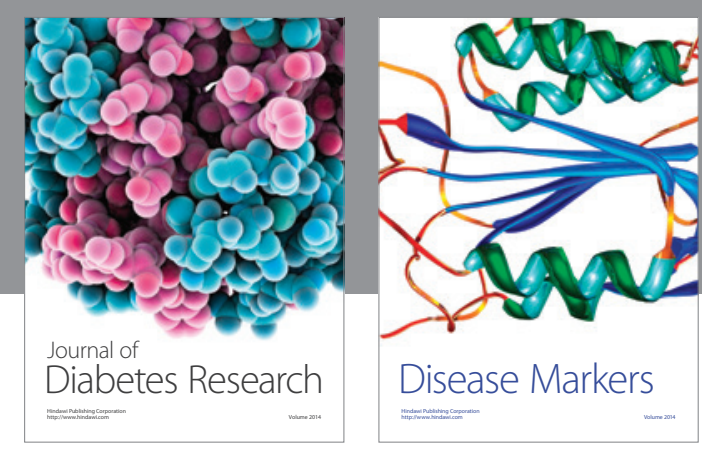

Disease Markers
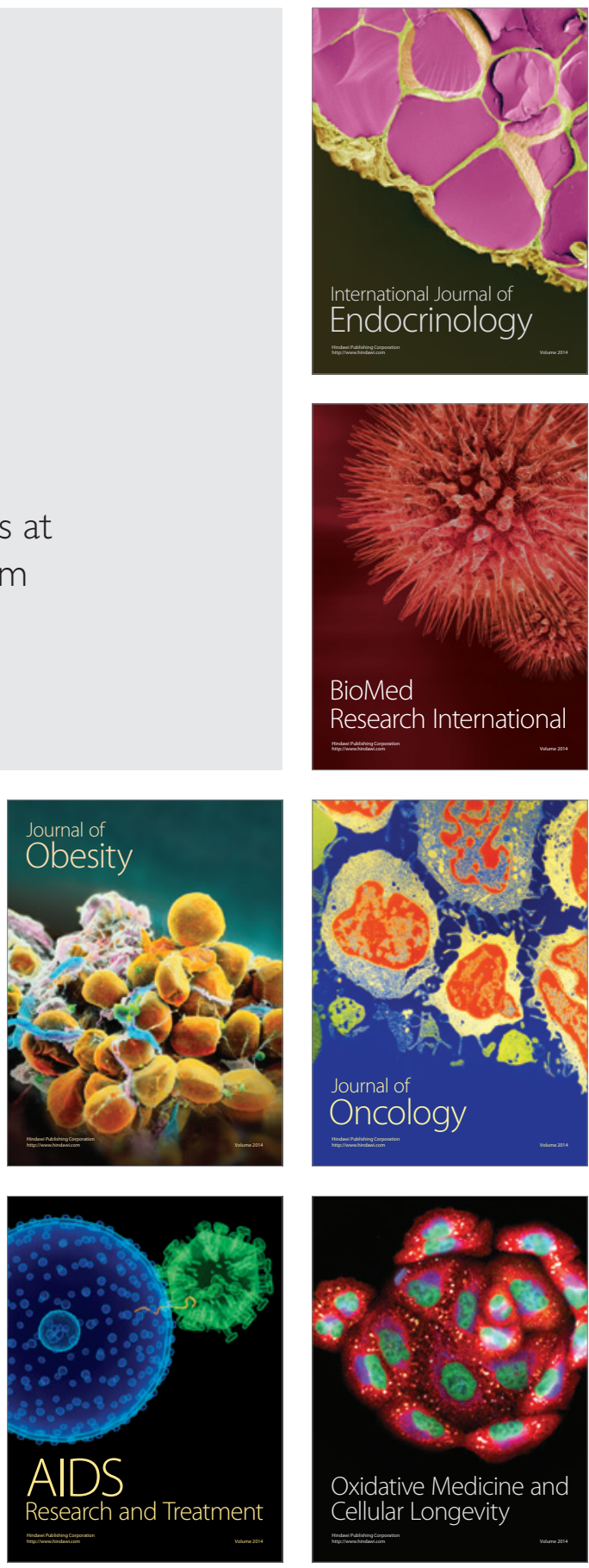